\title{
EFFECT OF SPATIAL ARRANGEMENTS ON YIELD AND COMPETITION OF JUTE (Corchorus olitorius L.) AND MUNGBEAN (Vigna radiata L. wilczek) IN INTERCROPPING
}

\author{
A.K. Chakraborty ${ }^{*}$, A.K. Ghorai, N.M. Alam, S. Roy and R. Saha \\ ICAR-Central Research Institute for Jute and Allied Fibres, Barrackpore \\ Kolkata, West Bengal
}

\begin{abstract}
Low profitability from jute farming, declining jute area and negligible pulse area are the rising concerns of farmers of Indo-Bangla subcontinent. This paper evaluated the extent of yield competition in jutemungbean intercropping with varying spatial geometry under alternate single row (SR) and double row (DR) planting. Two 2-year field experiments were conducted independently for the two systems of plantings involving jute ( $c v$ NJ 7010) and mungbean ( $c v$ TMB-37) arranged in systematic designs for $3 \times 3 \times 3$ spacing and plant density combinations with three replications. Yield competition was assessed using indices. Intercropping was found productive and profitable compared to sole cropping. Land equivalent ratios (LER) and area $\times$ time equivalent ratios (ATER) always exceeded unity. Jute equivalent yield (JEY) increased in the range of $4.9-45.3 \%$ and $30.7-51.1 \%$ over sole jute fibre yield and mean monetary advantage index exceeded 27100 and $31800 \mathrm{~F}^{\mathrm{h}} \mathrm{h}^{-1}$ for SR and DR planting, respectively. Economic advantage was higher for spacing combinations of $40 \mathrm{~cm}$ (row to row) $\times 6.5-8 \mathrm{~cm}$ (jute to jute in a row) $\times 10-12 \mathrm{~cm}$ (mungbean to mungbean in a row) in SR planting and for DR system it was at a band-to-band spacing of 11 $\mathrm{cm}$ with plant densities of $40-50 \mathrm{~m}^{-2}$ for jute and $25-30 \mathrm{~m}^{-2}$ for mungbean. Dense and intimate planting of jute reduced mung seed yield due to light stress. DR planting seemed more advantageous.
\end{abstract}

Keywords: Competition Indices, Corchorus olitorius, Jute-mungbean intercropping, Spatial arrangement, Systematic design, Vigna radiata

\section{INTRODUCTION}

Intercropping is the practice of growing two or more crops simultaneously on the same piece of land, where at least a portion of their respective production cycle overlap and crops are planted in sufficient closeness to offer competition for

\footnotetext{
* Corresponding Author: asim.chakraborty@icar.gov.in
} 
resources to each other (Hiebsch and McCollum, 1987). It is a prevalent practice among small holder subsistence farmers in tropical and sub-tropical latitudes (Dasgupta and Roy, 2016) and it can address the rising concerns on economic return, nutrition and climate change ( $\mathrm{Li}$ et al., 2019).

Jute, traditionally grown as rainfed sole crop, is the most affordable natural fibre and economically important crop of India and Bangladesh, sharing nearly similar agroclimatic conditions and small land holding (Mandal, 2016; George, 2015). Constraints in jute fibre profitability have led to its alarming area shrinkage during previous two decades in India (Kumari et al., 2018).

Extensive adaptation of legumes to several cropping systems has been studied widely (Li et al., 2019; Ofori and Stern, 1987). It is more suitable in low-input and laborintensive small-scale farming to ensure dependability of return in the event of crop loss, providing nutritious food and fodder and replenishing rich organic manure to soil (Altieri et al., 2012; Dasgupta and Roy, 2016; Rao and Willey, 1980). Shrinkage in acreages of these two crops started with the drive on production of more cereals (Dasgupta and Roy, 2016). Study on adaptation of mungbean in jute farming is emerging. India and Bangladesh are major importers of pulses with near-stagnated area and lesser possibility to increase in the future (Ali et al., 2012). Summer mungbean productivity of West Bengal, $\left(0.893 \mathrm{t} \mathrm{ha}^{-1}\right)$ and a potential seed yield of 0.7 to $1.0 \mathrm{t} \mathrm{ha}^{-1}$ is easily attainable in jute-mungbean intercropping (Ghorai et al., 2016). Recent advances in premature flowering resistant jute cultivars (Rahaman and Prasad, 2018) and development of short duration biotic stress resistant mungbean cultivars (Chadha, 2010) have opened the prospect of growing the crops together. In sole cropping, jute grows with a plant density of $35-50 \mathrm{~m}^{-2}$ and spacing of $25-30 \mathrm{~cm}$ $\times 7-8 \mathrm{~cm}$. Whereas, summer sole mungbean (March-May) is cultivated with a plant density of $25-30 \mathrm{~m}^{-2}$ and spacing of $25-30 \mathrm{~cm} \times 12-15 \mathrm{~cm}$ in West Bengal condition. As intercrop, mungbean effectively smother weeds in jute, reduce cost of weeding and add organic residue to soil (Ghorai et al., 2016).

Productivity and efficiency of intercropping system depends, largely on the spatial geometry of the component crops (Natarajan, 1990). For evaluation of the competition effects with spatial geometry and density (plants per unit area), experiments even with one species is challenging to randomization. The problem becomes more difficult with two or more crop species. To overcome the difficulties, systematic designs had been implemented (Snaydon, 1991; Mead, 1990; Natarajan, 1990; Thattil and Costa, 1988; Willey and Rao, 1980; Mead and Stern, 1980; Huxley and Maingu, 1978).

This study evaluated the nature and extent of competition of jute ( $c v \mathrm{NJ} 7010)$ and mungbean ( $c v$ TMB-37) in intercropping system under varied spatial arrangements. In a three-way systematic design, crops were planted in two types of row arrangements, (a) alternate single row (SR), i.e., 1:1 row system and (b) alternate double row (DR), i.e., 2:2 row system with14 $\mathrm{cm}$ wide two rows of either of the crops forming a narrow strip or a band. In this innovative study we aimed to achieve 
a reasonably full fibre yield as in sole jute, the main crop, along with some additional seed yield of mungbean. We hypothesize that information from this study will encourage to extensively integrate the practice of intercropping of mungbean with jute farming and will give impetus to study different aspects of jute-mungbean intercropping.

\section{MATERIALS AND METHODS}

\section{Experimental site}

The study was performed at the research farm of the Indian Council of Agricultural Research - Central Research Institute for Jute and Allied Fibres, Barrackpore, West Bengal, India $\left(22^{\circ} 45^{\prime} \mathrm{N}, 88^{\circ} 25^{\prime} \mathrm{E} ; 9.69 \mathrm{~m}\right.$ above mean sea level). The soil was Gangetic alluvium order inceptisol, having pH 7.23 (1:2.5 $\mathrm{w} / \mathrm{v}$ ), organic carbon $5.50 \mathrm{~g} \mathrm{~kg}^{-1}$, medium in fertility. The climate was humid tropical and it received an average annual rainfall of $1383.2 \mathrm{~mm}$. The trial was conducted between the third weeks of March to July of 2016 to 2018. Table 1 presents the weekly mean weather records of last 30 years (1989-2018) during the pre-monsoon summer months (March-May), coinciding with the production cycle of mungbean and rainfall distribution during 2016-2018. In 2016, the total rainfall was nearer to its long-term mean, with uneven distribution. In contrast, it was very low in 2017 and well distributed and excess in 2018.

Table 1. Long-term weekly weather record and rainfall distribution pattern of 201618 during the growing season of summer mungbean

\begin{tabular}{cccccccccc}
\hline $\begin{array}{c}\text { Standard } \\
\text { Meteorological } \\
\text { Week (SMW) }\end{array}$ & $\begin{array}{c}\text { Average of 30 years (1989-2018) } \\
\text { RH (\%) }\end{array}$ & $\begin{array}{c}\text { RH } \\
(\%) \\
(\text { morning) }\end{array}$ & $\begin{array}{c}\text { Max } \\
\text { Temp } \\
\left({ }^{\circ} \mathrm{C}\right)\end{array}$ & $\begin{array}{c}\text { Min } \\
\text { Temp } \\
\left({ }^{\circ} \mathrm{C}\right)\end{array}$ & $\begin{array}{c}\text { Rainfall } \\
(\mathrm{mm})\end{array}$ & $\begin{array}{c}\text { Rainfall } \\
(\mathrm{mm})\end{array}$ & $\begin{array}{c}\text { Rainfall } \\
(\mathrm{mm})\end{array}$ & $\begin{array}{c}\text { Rainfall } \\
(\mathrm{mm})\end{array}$ \\
\hline 10 & 92.8 & 42.5 & 31.9 & 18.1 & 1.9 & 0.0 & 23.8 & 0.0 \\
11 & 93.4 & 44.0 & 32.6 & 19.3 & 3.3 & 0.0 & 1.2 & 0.0 \\
12 & 93.3 & 46.6 & 34.0 & 21.5 & 9.9 & 24.0 & 2.4 & 0.0 \\
13 & 91.9 & 50.1 & 33.8 & 22.4 & 13.0 & 0.0 & 0.0 & 16.8 \\
14 & 91.6 & 50.9 & 34.5 & 23.2 & 8.7 & 0.0 & 1.0 & 44.4 \\
15 & 90.0 & 49.0 & 35.5 & 23.7 & 13.9 & 0.0 & 0.0 & 8.0 \\
16 & 89.9 & 52.5 & 35.8 & 24.9 & 8.9 & 0.0 & 0.0 & 29.2 \\
17 & 88.9 & 54.9 & 35.7 & 24.4 & 15.3 & 0.0 & 0.0 & 52.4 \\
18 & 88.0 & 56.8 & 35.3 & 24.8 & 21.8 & 9.8 & 13.2 & 57.0 \\
19 & 88.9 & 58.2 & 35.6 & 25.5 & 25.2 & 87.0 & 1.4 & 40.0 \\
20 & 89.7 & 60.7 & 35.5 & 25.0 & 32.0 & 76.4 & 4.4 & 41.2 \\
21 & 90.6 & 64.0 & 35.0 & 25.4 & 33.7 & 29.6 & 8.0 & 39.0 \\
22 & 89.4 & 62.1 & 35.2 & 26.2 & 46.9 & 34.4 & 91.2 & 84.2 \\
23 & 90.4 & 67.6 & 34.9 & 26.1 & 39 & 0.0 & 7.8 & 8 \\
Total & & & & & 273.5 & 261.2 & 154.4 & 420.2 \\
\hline
\end{tabular}




\section{Treatment combinations}

\section{Factorial treatment combinations for alternate single row planting (SR) and layout}

Let $\mathrm{I}, \mathrm{J}$ and $\mathrm{M}$ denote, respectively, the three factors, inter-row spacing $(\mathrm{cm})$, intrarow jute plant spacing $(\mathrm{cm})$ and intra-row mungbean plant spacing $(\mathrm{cm})$, each at 3 levels $(35,40,45),(5.0,6.5,8.0)$ and $(8,10,12)$, respectively. The $3 \times 3 \times 3$ factorial treatment combinations are $(35,5,8),(35,5,10),(35,5,12), \ldots,(45,8,12)$ and corresponded to $\mathrm{T}_{1}, \mathrm{~T}_{2}, \mathrm{~T}_{3}, \ldots, \mathrm{T}_{27}$, respectively. Table 2 listed the treatment combinations and the range of plant densities.

For a replicate, the layout took a typical form as in Figure 1(a). In a replicate, $18 \times 6.5$ $\mathrm{m}^{2}$ area was split breadthways into 3 segments of width $2.28,2.18$ and $2 \mathrm{~m}$, accommodated equal number of jute and mungbean rows in a segment, for systematically allocating the 3 levels of inter-row spacing $(35,40,45 \mathrm{~cm})$ in ascending or descending order. Within a segment, the 3 levels of intra-row jute spacing are arranged systematically in ascending or descending order, forming $3 \mathrm{sub}$ segments, each of length $6 \mathrm{~m}$. Again, within a $6 \mathrm{~m}$ long sub-segment receiving a particular level of intra-row jute spacing, 3 sub-sub-segments of length $2 \mathrm{~m}$ each, 3 intra-row mungbean spacing levels are allotted systematically in a manner such that the adjacent sub-segments within a segment looks a mirror image of the other for mungbean intra-row spacing combinations. Each factor of spacing varied independently of the other.

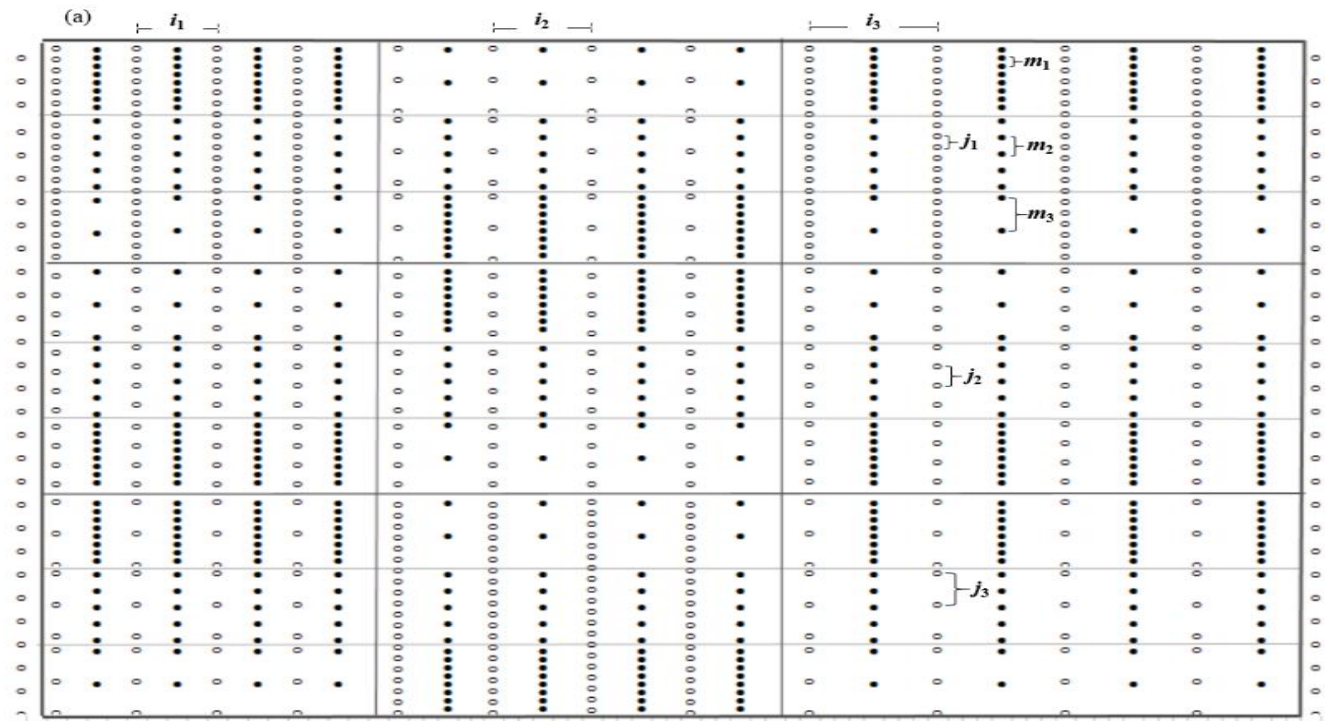




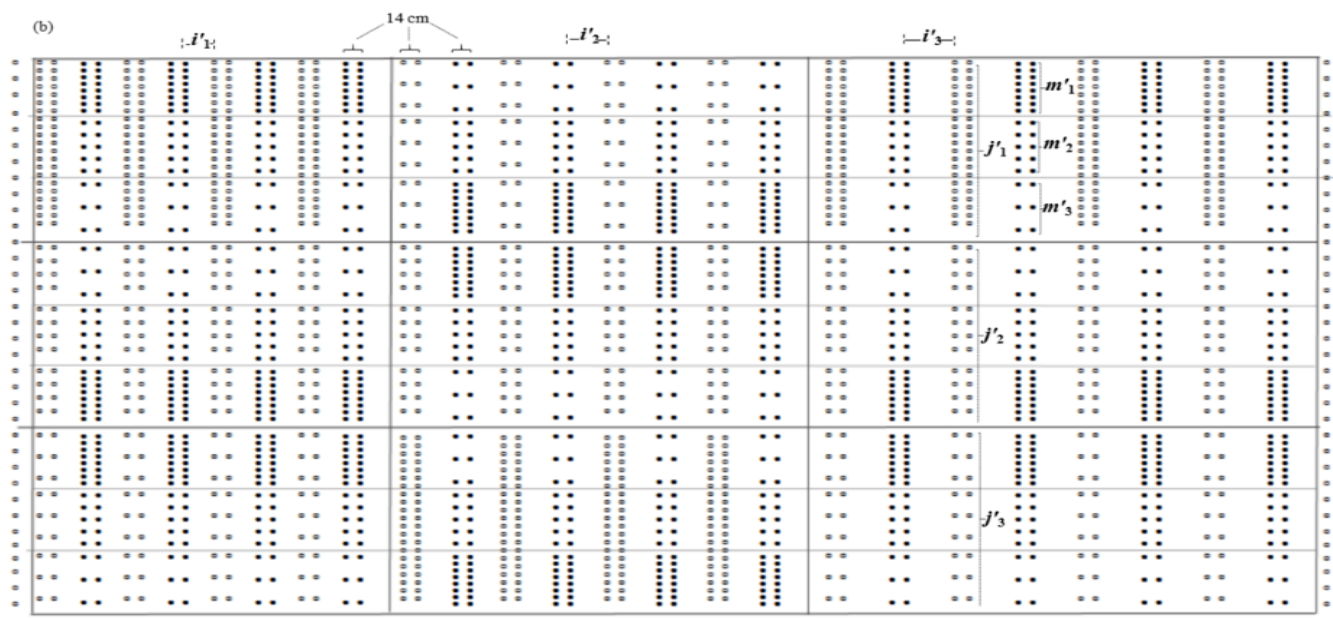

Figure 1. Layout of a three-way systematic design. $\circ$ : jute, ๑: mungbean. (a) Alternate single row (SR) arrangement: $i_{\mathrm{s}}=$ inter-row spacing; $j_{\mathrm{t}}=$ intrarow spacing of jute; $\mathrm{m}_{\mathrm{u}}=$ intra-row spacing of mungbean; (b) Alternate double row (DR) arrangement, $14 \mathrm{~cm}$ wide two rows form a narrow strip or a band. Factor levels: $i_{\mathrm{S}}=$ inter-band spacing; $\mathrm{j}_{\mathrm{t}}=$ jute plant density; $\mathrm{m}_{\mathrm{u}}{ }_{\mathrm{u}}=$ mungbean plant density, where $\mathrm{s}, \mathrm{t}, \mathrm{u}=1,2,3$.

Factorial treatment combinations for the alternate double row planting (DR) and its layout

LetI', J' and M'denote, respectively, the 3 factors of inter-band spacing $(\mathrm{cm})$, jute plant density $\left(\mathrm{ha}^{-1}\right)$ and mungbean plant density $\left(\mathrm{ha}^{-1}\right)$ each at 3 levels $(11,14,17),(3,4,5)$ and $(2,2.5,3)$. The $3 \times 3 \times 3$ factorial treatment combinations are $(11,3,2),(11,3,2.5)$, $(11,3,3), \ldots,(17,5,3)$, corresponded to $\mathrm{T}_{1}^{\prime}, \mathrm{T}_{2}^{\prime}, \mathrm{T}_{3}^{\prime}, \ldots \mathrm{T}_{27}^{\prime}$. Table 3 listed the treatment combinations and the range of intra-plant spacing combinations.

For a replicate, the layout for DR system took a typical form as in Figure 1(b). Itis similar to that of SR, except the inter-band spacing and plant density respectively replaced the factors of inter-row spacing and intra-plant spacing of component crops in SR.

\section{Management of crops and data recording}

A basal fertilizer dose (10:26:26 of N:P:K at the rate of $\left.250 \mathrm{~kg} \mathrm{ha}^{-1}\right)$ was applied during sowing. Jute ( $c v$ NJ 7010) and mungbean ( $c v$ TMB-37) seeds were line sown in East-West direction, at 2-3 cm and 4-6 cm soil depth, respectively, on the third week of March and was irrigated $(50 \mathrm{~mm})$. Designed spacing combinations or plant stands were maintained after final thinning. Sole jute plot was top dressed with $\mathrm{N}$ fertilizer at the rate of $20 \mathrm{~kg} \mathrm{ha}^{-1}$ at 21 DAS and 40 DAS. Top dressings of $\mathrm{N}$ fertilizer were withheld for intercropped plots until the final pod-picking day. 
Table 2. Treatment combinations with inter-row spacing and within-row plant spacing levels, corresponding plant densities, biological yield of jute fibre and mungbean seed, jute equivalent yield (JEY), land equivalent ratio $(\mathrm{LER})$, area $\times$ time equivalent ratio (ATER), relative crowding coefficient $(\mathrm{K})$, aggressivity $(\mathrm{A})$ and monetary advantage indices (MAI) in jute-mungbean intercropping system under alternate single row (SR) planting.

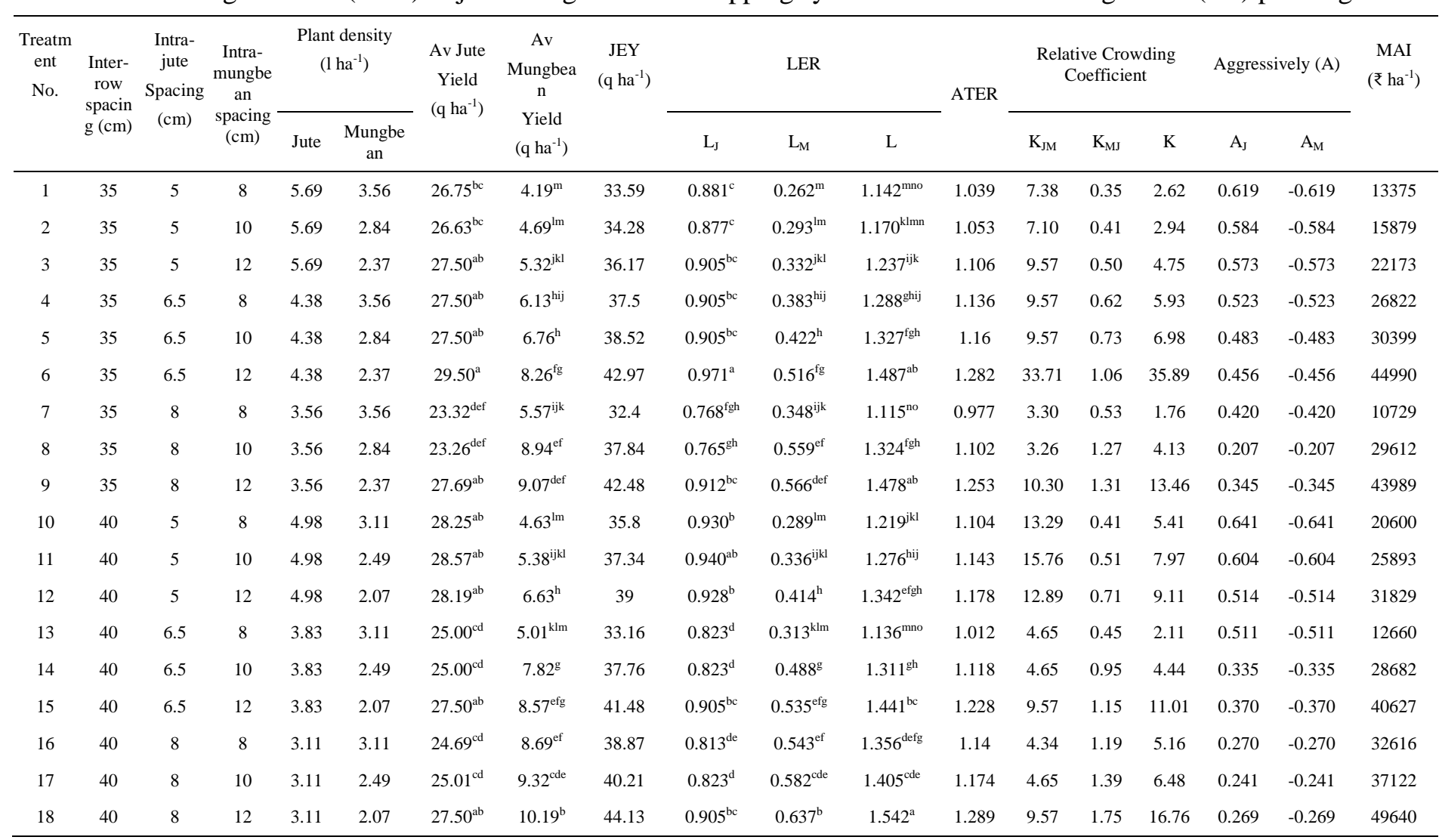




\begin{tabular}{|c|c|c|c|c|c|c|c|c|c|c|c|c|c|c|c|c|c|c|}
\hline \multirow[t]{2}{*}{$\begin{array}{c}\text { Treatm } \\
\text { ent } \\
\text { No. }\end{array}$} & \multirow{2}{*}{$\begin{array}{l}\text { Inter- } \\
\text { row } \\
\text { spacin } \\
\mathrm{g}(\mathrm{cm})\end{array}$} & \multirow{2}{*}{$\begin{array}{c}\begin{array}{c}\text { Intra- } \\
\text { jute }\end{array} \\
\text { Spacing } \\
(\mathrm{cm})\end{array}$} & \multirow{2}{*}{$\begin{array}{l}\text { Intra- } \\
\text { mungbe } \\
\text { an } \\
\text { spacing } \\
(\mathrm{cm})\end{array}$} & \multicolumn{2}{|c|}{$\begin{array}{l}\text { Plant density } \\
\qquad\left(1 \mathrm{ha}^{-1}\right)\end{array}$} & \multirow{2}{*}{$\begin{array}{l}\text { Av Jute } \\
\text { Yield } \\
\left(\mathrm{q} \mathrm{ha}^{-1}\right)\end{array}$} & \multirow{2}{*}{$\begin{array}{c}\mathrm{Av} \\
\text { Mungbea } \\
\mathrm{n} \\
\text { Yield }^{-} \\
\left(\mathrm{q} \mathrm{ha}^{-1}\right)\end{array}$} & \multirow[t]{2}{*}{$\begin{array}{c}\mathrm{JEY} \\
\left(\mathrm{q} \mathrm{ha}^{-1}\right)\end{array}$} & \multicolumn{3}{|c|}{ LER } & \multirow{2}{*}{ ATER } & \multicolumn{3}{|c|}{$\begin{array}{l}\text { Relative Crowding } \\
\text { Coefficient }\end{array}$} & \multicolumn{2}{|c|}{ Aggressively (A) } & \multirow[t]{2}{*}{$\begin{array}{l}\text { MAI } \\
\left(₹ \mathrm{ha}^{-1}\right)\end{array}$} \\
\hline & & & & Jute & $\begin{array}{c}\text { Mungbe } \\
\text { an }\end{array}$ & & & & $\mathrm{L}_{\mathrm{J}}$ & $\mathrm{L}_{\mathrm{M}}$ & $\mathrm{L}$ & & $\mathrm{K}_{\mathrm{JM}}$ & $\mathrm{K}_{\mathrm{MJ}}$ & K & $\mathrm{A}_{\mathrm{J}}$ & $\mathrm{A}_{\mathrm{M}}$ & \\
\hline 19 & 45 & 5 & 8 & 4.42 & 2.76 & $23.00^{\text {def }}$ & $5.44^{\mathrm{ijkl}}$ & 31.88 & $0.757^{\mathrm{gh}}$ & $0.340^{\mathrm{ijkl}}$ & $1.097^{\circ}$ & 0.962 & 3.12 & 0.51 & 1.61 & 0.417 & -0.417 & 9012 \\
\hline 20 & 45 & 5 & 10 & 4.42 & 2.21 & $23.38^{\text {def }}$ & $6.07^{\text {hij }}$ & 33.27 & $0.770^{\mathrm{fgh}}$ & $0.379^{\mathrm{hij}}$ & $1.149^{\mathrm{gmno}}$ & 0.998 & 3.34 & 0.61 & 2.04 & 0.391 & -0.391 & 13733 \\
\hline 21 & 45 & 5 & 12 & 4.42 & 1.84 & $24.69^{\mathrm{cd}}$ & $9.88^{\text {bcd }}$ & 40.81 & $0.813^{\mathrm{de}}$ & $0.617^{\mathrm{bcd}}$ & $1.430^{\mathrm{bc}}$ & 1.185 & 4.34 & 1.61 & 7.00 & 0.196 & -0.196 & 39268 \\
\hline 22 & 45 & 6.5 & 8 & 3.4 & 2.76 & $23.44^{\text {def }}$ & $6.19^{\text {hi }}$ & 33.54 & $0.772^{\mathrm{fg}}$ & $0.387^{\mathrm{hi}}$ & $1.158^{\operatorname{lmno}}$ & 1.005 & 3.38 & 0.63 & 2.13 & 0.385 & -0.385 & 14662 \\
\hline 23 & 45 & 6.5 & 10 & 3.4 & 2.21 & $23.57^{\mathrm{def}}$ & $6.76^{\mathrm{h}}$ & 34.58 & $0.776^{\mathrm{efg}}$ & $0.422^{\mathrm{h}}$ & $1.198^{\mathrm{klm}}$ & 1.03 & 3.46 & 0.73 & 2.52 & 0.354 & -0.354 & 18239 \\
\hline 24 & 45 & 6.5 & 12 & 3.4 & 1.84 & $24.44^{\text {cde }}$ & $6.63^{\mathrm{h}}$ & 35.25 & $0.805^{\mathrm{def}}$ & $0.414^{\mathrm{h}}$ & $1.219^{\mathrm{jkl}}$ & 1.054 & 4.12 & 0.71 & 2.91 & 0.390 & -0.390 & 20242 \\
\hline 25 & 45 & 8 & 8 & 2.76 & 2.76 & $23.13^{\text {def }}$ & $10.07^{\mathrm{bc}}$ & 39.56 & $0.761^{\mathrm{gh}}$ & $0.629^{\mathrm{bc}}$ & $1.390^{\text {cdef }}$ & 1.141 & 3.19 & 1.69 & 5.41 & 0.132 & -0.132 & 35549 \\
\hline 26 & 45 & 8 & 10 & 2.76 & 2.21 & $21.38^{\mathrm{f}}$ & $11.44^{\mathrm{a}}$ & 40.05 & $0.704^{i}$ & $0.715^{\mathrm{a}}$ & $1.419^{\mathrm{bcd}}$ & 1.135 & 2.38 & 2.51 & 5.95 & -0.011 & 0.011 & 37838 \\
\hline 27 & 45 & 8 & 12 & 2.76 & 1.84 & $22.25^{\mathrm{ef}}$ & $9.07^{\text {def }}$ & 37.05 & $0.733^{\mathrm{hi}}$ & $0.566^{\text {def }}$ & $1.299^{\mathrm{ghi}}$ & 1.074 & 2.74 & 1.31 & 3.58 & 0.166 & -0.166 & 27252 \\
\hline Mean & & & & & & 25.5 & 7.28 & 37.39 & 0.840 & 0.455 & 1.295 & 1.114 & 7.53 & 0.95 & 6.67 & 0.385 & -0.385 & 27180 \\
\hline $\mathrm{CV}$ & & & & & & 9.61 & 29.19 & & & & & & & & & & & \\
\hline
\end{tabular}

Mean followed by same letter are not significantly different based on DMRT $(\mathrm{p}=0.05)$ 
Table 3. Treatments with Inter-band spacing and plant densities of jute and mungbean, corresponding within-row plant spacing levels, biological yield of jute fibre and mungbean seed, jute equivalent yield (JEY), land equivalent ratio (LER), area $\times$ time equivalent ratio (ATER), relative crowding coefficient (K), aggressivity (A) and monetary advantage indices (MAI) in jute-mungbean intercropping system under alternate double row (DR) planting.

\begin{tabular}{|c|c|c|c|c|c|c|c|c|c|c|c|c|c|c|c|c|c|c|}
\hline \multirow{2}{*}{$\begin{array}{c}\text { Treatm } \\
\text { ent } \\
\text { No. }\end{array}$} & \multirow{2}{*}{$\begin{array}{l}\text { Inter- } \\
\text { band } \\
\text { Spaci } \\
\text { ng } \\
(\mathrm{cm})\end{array}$} & \multicolumn{2}{|c|}{$\begin{array}{l}\text { Plant Density } \\
\qquad\left(1 \mathrm{ha}^{-1}\right)\end{array}$} & \multirow{2}{*}{$\begin{array}{c}\text { Intra- } \\
\text { jute } \\
- \text { Spaci } \\
\text { ng } \\
(\mathrm{cm})\end{array}$} & \multirow{2}{*}{$\begin{array}{c}\text { Intra- } \\
\text { mungbea } \\
\mathrm{n} \\
\text { spacing } \\
(\mathrm{cm})\end{array}$} & \multirow{2}{*}{$\begin{array}{c}\text { Av Jute } \\
\text { Yield } \\
\left(\mathrm{q} \mathrm{ha}^{-1}\right)\end{array}$} & \multirow{2}{*}{$\begin{array}{c}\mathrm{Av} \\
\text { Mungbean } \\
\text { Yield } \\
\left(\mathrm{q} \mathrm{ha}^{-1}\right)\end{array}$} & \multirow{2}{*}{$\begin{array}{c}\text { JEY } \\
\left(\mathrm{q} \mathrm{ha}^{-1}\right)\end{array}$} & \multicolumn{3}{|c|}{ LER } & \multirow{2}{*}{ ATER } & \multicolumn{3}{|c|}{$\begin{array}{l}\text { Relative crowding } \\
\text { coefficient }\end{array}$} & \multicolumn{2}{|c|}{$\begin{array}{l}\text { Aggressively } \\
\text { (A) }\end{array}$} & \multirow{2}{*}{$\begin{array}{l}\text { MAI } \\
\left(₹ h^{-1}\right.\end{array}$} \\
\hline & & Jute & $\begin{array}{c}\text { Mungb } \\
\text { ean }\end{array}$ & & & & & & $\mathrm{L}_{\mathrm{J}}$ & $\mathrm{L}_{\mathrm{M}}$ & $\mathrm{L}$ & & $\mathrm{K}_{\mathrm{JM}}$ & $\mathrm{K}_{\mathrm{MJ}}$ & K & $\mathrm{A}_{J}$ & $\mathrm{~A}_{\mathrm{M}}$ & \\
\hline 1 & 11 & 3 & 2 & 14.3 & 21.5 & $16.94^{\mathrm{d}}$ & $10.13^{\mathrm{ab}}$ & 36.02 & $0.652^{\mathrm{j}}$ & $0.633^{\mathrm{ab}}$ & $1.284^{\mathrm{ab}}$ & 1.04 & 1.87 & 1.72 & 3.22 & 0.04 & -0.04 & 29469 \\
\hline 2 & 11 & 3 & 2.5 & 14.3 & 17.2 & $17.13^{\text {cd }}$ & $10.32^{\mathrm{a}}$ & 36.57 & $0.659^{\mathrm{ij}}$ & $0.645^{\mathrm{a}}$ & $1.303^{\mathrm{ab}}$ & 1.055 & 1.93 & 1.81 & 3.50 & 0.03 & -0.03 & 31472 \\
\hline 5 & 11 & 4 & 2.5 & 10.8 & 17.2 & $20.57^{\text {abcd }}$ & $9.94^{\mathrm{abc}}$ & 39.3 & $0.791^{\text {abcdefg }}$ & $0.621^{\mathrm{abc}}$ & $1.412^{\mathrm{a}}$ & 1.173 & 3.78 & 1.64 & 6.20 & 0.34 & -0.34 & 42415 \\
\hline 6 & 11 & 4 & 3 & 10.8 & 14.3 & $20.26^{\text {abcd }}$ & $9.44^{\mathrm{abc}}$ & 38.04 & $0.779^{\text {bcdefg }}$ & $0.590^{\text {abcd }}$ & $1.369^{\mathrm{ab}}$ & 1.141 & 3.52 & 1.44 & 5.06 & 0.38 & -0.38 & 37909 \\
\hline 7 & 11 & 5 & 2 & 8.6 & 21.5 & $20.07^{\text {abcd }}$ & $9.7 \mathrm{a}^{\mathrm{abc}}$ & 38.44 & $0.772^{\text {cdefgh }}$ & $0.609^{\mathrm{abc}}$ & $1.381^{\mathrm{ab}}$ & 1.146 & 3.38 & 1.56 & 5.27 & 0.32 & -0.32 & 39268 \\
\hline 8 & 11 & 5 & 2.5 & 8.6 & 17.2 & $20.44^{\text {abcd }}$ & $9.38^{\mathrm{abc}}$ & 38.11 & $0.786^{\text {bcdefg }}$ & $0.586^{\text {abcde }}$ & $1.372^{\mathrm{ab}}$ & 1.146 & 3.67 & 1.42 & 5.20 & 0.40 & -0.40 & 38267 \\
\hline 9 & 11 & 5 & 3 & 8.6 & 14.3 & $19.88^{\text {abcd }}$ & $9.58^{\mathrm{abc}}$ & 37.93 & $0.764^{\text {cdefgh }}$ & $0.598^{\text {abcd }}$ & $1.363^{\mathrm{ab}}$ & 1.132 & 3.24 & 1.49 & 4.84 & 0.33 & -0.33 & 37337 \\
\hline 14 & 14 & 4 & 2.5 & 9.5 & 15.2 & $21.19^{\text {abcd }}$ & $8.19^{\mathrm{abc}}$ & 36.62 & $0.815^{\text {abcdefg }}$ & $0.512^{\text {cdefghij }}$ & $1.327^{\mathrm{ab}}$ & 1.129 & 4.40 & 1.05 & 4.61 & 0.61 & -0.61 & 33331 \\
\hline 15 & 14 & 4 & 3 & 9.5 & 12.7 & $20.57^{\text {abcd }}$ & $7.57^{\mathrm{abc}}$ & 34.82 & $0.791^{\text {abcdefg }}$ & $0.473^{\text {fghijk }}$ & $1.264^{\mathrm{ab}}$ & 1.081 & 3.78 & 0.90 & 3.39 & 0.64 & -0.64 & 26894 \\
\hline 16 & 14 & 5 & 2 & 7.6 & 19.0 & $21.75^{\text {abcd }}$ & $7.38^{\mathrm{abc}}$ & 35.65 & $0.837^{\mathrm{abcd}}$ & $0.461^{\text {fghijk }}$ & $1.298^{\mathrm{ab}}$ & 1.12 & 5.12 & 0.86 & 4.38 & 0.75 & -0.75 & 30256 \\
\hline 17 & 14 & 5 & 2.5 & 7.6 & 15.2 & $22.88^{\mathrm{ab}}$ & $6.57^{\mathrm{bc}}$ & 35.25 & $0.880^{\mathrm{ab}}$ & $0.410^{\mathrm{jk}}$ & $1.290^{\mathrm{ab}}$ & 1.132 & 7.32 & 0.70 & 5.09 & 0.94 & -0.94 & 29326 \\
\hline 18 & 14 & 5 & 3 & 7.6 & 12.7 & $21.51^{\mathrm{abcd}}$ & $6.63^{\mathrm{bc}}$ & 33.99 & $0.827^{\text {abcdef }}$ & $0.414^{\mathrm{ijk}}$ & $1.241^{\mathrm{b}}$ & 1.081 & 4.78 & 0.71 & 3.38 & 0.83 & -0.83 & 24391 \\
\hline 19 & 17 & 3 & 2 & 11.6 & 17.4 & $18.94^{\text {abcd }}$ & $7.94^{\mathrm{abc}}$ & 37.68 & $0.777^{\text {bcdefgh }}$ & $0.496^{\text {defghijk }}$ & $1.273^{\mathrm{ab}}$ & 1.179 & 6.92 & 0.98 & 6.81 & 0.76 & -0.76 & 37623 \\
\hline 20 & 17 & 3 & 2.5 & 11.6 & 14.0 & $18.82^{\text {abcd }}$ & $8.38^{\text {abc }}$ & 34.07 & $0.728^{\text {efghij }}$ & $0.523^{\text {bcdefghi }}$ & $1.252^{\mathrm{b}}$ & 1.025 & 2.37 & 1.10 & 2.60 & 0.36 & -0.36 & 23246 \\
\hline
\end{tabular}




\begin{tabular}{|c|c|c|c|c|c|c|c|c|c|c|c|c|c|c|c|c|c|c|}
\hline \multirow{2}{*}{$\begin{array}{c}\text { Treatm } \\
\text { ent } \\
\text { No. }\end{array}$} & \multirow{2}{*}{$\begin{array}{c}\text { Inter- } \\
\text { band } \\
\text { Spaci } \\
\text { ng } \\
(\mathrm{cm})\end{array}$} & \multicolumn{2}{|c|}{$\begin{array}{c}\text { Plant Density } \\
\left(1 \text { ha }^{-1}\right) \\
\end{array}$} & \multirow{2}{*}{$\begin{array}{l}\text { Intra- } \\
\text { jute } \\
\text { Spaci } \\
\text { ng } \\
(\mathrm{cm})\end{array}$} & \multirow{2}{*}{$\begin{array}{c}\text { Intra- } \\
\text { mungbea } \\
n \\
\text { spacing } \\
(\mathrm{cm})\end{array}$} & \multirow{2}{*}{$\begin{array}{c}\text { Av Jute } \\
\text { Yield } \\
\left(\mathrm{q} \mathrm{ha}^{-1}\right)\end{array}$} & \multirow{2}{*}{$\begin{array}{c}\text { Av } \\
\text { Mungbean } \\
\text { Yield } \\
\left(\mathrm{q} \mathrm{ha}^{-1}\right)\end{array}$} & \multirow{2}{*}{$\begin{array}{l}\mathrm{JEY} \\
\left(\mathrm{q} \mathrm{ha}^{-1}\right)\end{array}$} & \multicolumn{3}{|c|}{ LER } & \multirow[b]{2}{*}{ ATER } & \multicolumn{3}{|c|}{$\begin{array}{c}\text { Relative crowding } \\
\text { coefficient }\end{array}$} & \multicolumn{2}{|c|}{$\begin{array}{l}\text { Aggressively } \\
\text { (A) }\end{array}$} & \multirow{2}{*}{$\begin{array}{l}\text { MAI } \\
\left(₹ \mathrm{ha}^{-1}\right)\end{array}$} \\
\hline & & Jute & $\begin{array}{l}\text { Mungb } \\
\text { ean }\end{array}$ & & & & & & $\mathrm{L}_{\mathrm{J}}$ & $\mathrm{L}_{\mathrm{M}}$ & $\mathrm{L}$ & & $\mathrm{K}_{\mathrm{JM}}$ & $\mathrm{K}_{\mathrm{MJ}}$ & K & $\mathrm{A}_{\mathrm{J}}$ & $\mathrm{A}_{\mathrm{M}}$ & \\
\hline 21 & 17 & 3 & 3 & 11.6 & 11.6 & $21.57^{\text {abcd }}$ & $9.07^{\mathrm{abc}}$ & 35.9 & $0.724^{\text {fghij }}$ & $0.566^{\text {abcdef }}$ & $1.290^{\mathrm{ab}}$ & 1.072 & 2.62 & 1.31 & 3.42 & 0.31 & -0.31 & 29827 \\
\hline 22 & 17 & 4 & 2 & 8.7 & 17.4 & $21.63^{\text {abcd }}$ & $8.63^{\mathrm{abc}}$ & 37.82 & $0.829^{\text {abcde }}$ & $0.533^{\text {abcdefgh }}$ & $1.369^{\mathrm{ab}}$ & 1.161 & 4.86 & 1.17 & 5.68 & 0.58 & -0.58 & 37695 \\
\hline 23 & 17 & 4 & 2.5 & 8.7 & 14.0 & $19.88^{\text {abcd }}$ & $7.25^{\mathrm{abc}}$ & 35.29 & $0.832^{\text {abcde }}$ & $0.453^{\text {ghijk }}$ & $1.285^{\mathrm{ab}}$ & 1.11 & 4.94 & 0.83 & 4.10 & 0.76 & -0.76 & 28968 \\
\hline 25 & 17 & 5 & 2 & 7.0 & 17.4 & $23.19^{\mathrm{a}}$ & $6.38^{\mathrm{c}}$ & 34.89 & $0.880^{\mathrm{ab}}$ & $0.399^{k}$ & $1.279^{\mathrm{ab}}$ & 1.125 & 7.32 & 0.66 & 4.85 & 0.96 & -0.96 & 28110 \\
\hline 26 & 17 & 5 & 2.5 & 7.0 & 14.0 & $22.44^{\mathrm{abc}}$ & $7.07^{\mathrm{abc}}$ & 36.5 & $0.892^{\mathrm{a}}$ & $0.441^{\mathrm{hijk}}$ & $1.333^{\mathrm{ab}}$ & 1.163 & 8.24 & 0.79 & 6.51 & 0.90 & -0.90 & 33761 \\
\hline 27 & 17 & 5 & 3 & 7.0 & 11.6 & $16.94^{\mathrm{d}}$ & $7.26^{\mathrm{abc}}$ & 36.1 & $0.863^{\mathrm{abc}}$ & $0.453^{\text {ghijk }}$ & $1.317^{\mathrm{ab}}$ & 1.142 & 6.30 & 0.83 & 5.22 & 0.82 & -0.82 & 32115 \\
\hline Mean & & & & & & 20.22 & 8.45 & 36.3 & 0.863 & 0.453 & 1.311 & 1.106 & 4.06 & 1.18 & 4.36 & 0.50 & -0.50 & 31829 \\
\hline CV & & & & & & 12.13 & 20.28 & & & & & & & & & & & \\
\hline
\end{tabular}

Mean followed by same letter are not significantly different based on DMRT $(\mathrm{p}=0.05)$ 
Adequate plant protection measures were taken for mungbean. Mungbean pods were picked in 3 dates in between 55-75 DAS. After final pod harvest, mungbean plants were left in the field for in-situ decomposition and jute plants were harvested at 120 DAS. Crop yields, plant height, pod count and light flux reading were recorded. Calculation of competition indices were done separately for SR and DR systems.

\section{Competition indices}

Competition in jute-mungbean intercropping systems were assessed using indices of land equivalent ratio (Willey, 1979), area $\times$ time equivalency ratio (Hiebsch and McCollum, 1987), jute equivalent yield (Lal and Ray, 1976), relative crowding coefficient (de Wit, 1960; Willey, 1979), coefficient of aggressivity (McGilchrist, 1965; Willey and Rao, 1980), and monetary advantage index (Ali and Mishra, 1993).

\section{Land equivalent ratio (LER)}

LER is defined as

$$
\mathrm{LER}=\mathrm{L}_{\mathrm{J}}+\mathrm{L}_{\mathrm{M}} ; \mathrm{L}_{\mathrm{J}}=\mathrm{LER} \text { of jute }=\mathrm{Y}_{\mathrm{JI}} / \mathrm{Y}_{\mathrm{JS}} \text { and } \mathrm{L}_{\mathrm{M}}=\mathrm{LER} \text { of mungbean }=\mathrm{Y}_{\mathrm{MI}} / \mathrm{Y}_{\mathrm{MS}}
$$

Where, $\mathrm{Y}_{\mathrm{JI}}$ and $\mathrm{Y}_{\mathrm{MI}}$ are the yields of jute fibre and mungbean seed in intercropping; and $\mathrm{Y}_{\mathrm{JS}}$ and $\mathrm{Y}_{\mathrm{MS}}$ are the corresponding yields in sole cropping, respectively.

\section{Area $\times$ time equivalency ratio (ATER)}

ATER is the weighted sum of partial LERs of the component crops, weights being the proportionate production cycle days of the corresponding component crops. It is defined as

$$
\operatorname{ATER}=\left(\mathrm{t}_{\mathrm{J}} \times \mathrm{L}_{\mathrm{J}}+\mathrm{t}_{\mathrm{M}} \times \mathrm{L}_{\mathrm{M}}\right) / \max \left(\mathrm{t}_{\mathrm{J}}, \mathrm{t}_{\mathrm{M}}\right)
$$

Where, $t_{J}$ and $t_{M}$ are the duration of production cycle (days) for jute and mungbean, respectively and $\max \left(\mathrm{t}_{\mathrm{J}}, \mathrm{t}_{\mathrm{M}}\right)$ is the maximum duration of an intercropping system (days).

\section{Jute equivalent yield (JEY)}

JEY converts mungbean seed yield to jute equivalent yield in terms of the prevailing price. In intercropping condition, JEY is calculated using the formulae:

$$
\mathrm{JEY}=\mathrm{Y}_{\mathrm{JI}}+\mathrm{Y}_{\mathrm{MI}} \times\left(\mathrm{P}_{\mathrm{M}} / \mathrm{P}_{\mathrm{J}}\right)
$$

Where, $\mathrm{P}_{\mathrm{J}}$ and $\mathrm{P}_{\mathrm{M}}$ are the price $\left(₹ \mathrm{q}^{-1}\right)$ of jute and mungbean, respectively, during the time of the study.

\section{Relative crowding coefficient $(\mathrm{K})$}

The coefficient $\mathrm{K}$ is calculated by

$$
\mathrm{K}=\mathrm{K}_{\mathrm{JM}} \times \mathrm{K}_{\mathrm{MJ}}
$$

Where, $\mathrm{K}_{\mathrm{JM}}$ and $\mathrm{K}_{\mathrm{MJ}}$ are the relative crowding coefficients of jute intercropped with mungbean and mungbean intercropped with jute, respectively and measured using the formulae 


$$
\mathrm{K}_{\mathrm{JM}}=\frac{\mathrm{Y}_{\mathrm{JI}} \times \mathrm{Z}_{\mathrm{JM}}}{\left(\mathrm{Y}_{\mathrm{JS}}-\mathrm{Y}_{\mathrm{JI}}\right) \times \mathrm{Z}_{\mathrm{MJ}}} \text { and } \mathrm{K}_{\mathrm{MJ}}=\frac{\mathrm{Y}_{\mathrm{MI}} \times \mathrm{Z}_{\mathrm{MJ}}}{\left(\mathrm{Y}_{\mathrm{MS}}-\mathrm{Y}_{\mathrm{MI}}\right) \times \mathrm{Z}_{\mathrm{JM}}}
$$

Where, $\mathrm{Z}_{\mathrm{JM}}$ andZ $\mathrm{Z}_{\mathrm{MJ}}$ are the sown proportion of jute and mungbean in intercropping, respectively.

\section{Coefficient of aggressivity (A)}

It measures how much the relative yield of one crop is greater than that of the other by the formulae

$$
\mathrm{A}_{\mathrm{J}}=\left(\mathrm{L}_{\mathrm{J}} / \mathrm{Z}_{\mathrm{JI}}\right)-\left(\mathrm{L}_{\mathrm{M}} / \mathrm{Z}_{\mathrm{MI}}\right) \text { and } \mathrm{A}_{\mathrm{M}}=\left(\mathrm{L}_{\mathrm{M}} / \mathrm{Z}_{\mathrm{MI}}\right)-\left(\mathrm{L}_{\mathrm{J}} / \mathrm{Z}_{\mathrm{JI}}\right)
$$

Where, $A_{J}$ and $A_{M}$ are partial aggressivity coefficients of jute and mungbean and $Z_{\mathrm{JI}}$, $\mathrm{Z}_{\mathrm{MI}}$ are the sown proportion of jute and mungbean in intercropping, respectively.

\section{Monetary advantage index (MAI)}

MAI provides information on the economic advantage of the intercropping system and calculated as

MAI = Value of combined intercrop yield $\times($ LER -1$) /$ LER

\section{Statistical Analysis}

A conventional analysis of variance (ANOVA) may not be valid for systematic designs, since many effects have been confounded due to systematic arrangement and restricted randomization of treatment combinations. Nevertheless, an ANOVA can be used as a preliminary diagnostic tool to give some indication of the significance or otherwise of the effects of the factors and their interactions (Willey and Rao, 1980; Thattil and Costa, 1988). In the present study, ANOVA was performed on yield data and LER values. Student t-test and Duncan's multiple range tests (DMRT) were performed to test mean differences using SAS 9.3 software.

\section{RESULTS AND DISCUSSION}

\section{Yield attributing characters}

There was no significant change in jute plant height due to intercropping. In contrast, a significant $(\mathrm{p}<0.05)$ increase in average mungbean plant height was observed at 55 DAS with $29.6 \mathrm{~cm}$ in sole crop and $37 \mathrm{~cm}$ in SR, whereas it was $45.6 \mathrm{~cm}$ in sole crop and $51.33 \mathrm{~cm}$ in DR. At 45 DAS, jute canopy caused 6\% to $23.66 \%$ reduction in solar radiation on mungbean canopy at $10: 30 \mathrm{~h}$ with a mean reduction of $13.07 \%$; whereas, at $12: 30 \mathrm{~h}$, the mean reduction was $2.45 \%$. The three stages of pod picking contributed about $30 \%, 50 \%$ and $15 \%$ of the total seed yield, respectively. Results for DR planting were straightforward in comparison to SR. Shading from taller jute plant canopy may have induced elongation of mungbean plants and affected seed yield. These results conformed to earlier studies on mungbean plant height and shading effect (Roy et al., 2015). 


\section{Comparative yield advantages}

In the Analysis of Variance for SR planting, all the main effects and 2- and 3-factor interactions were significant $(\mathrm{p}<0.05)$ on jute fibre yield, mungbean seed yield, $\mathrm{L}_{\mathrm{J}}$, $\mathrm{L}_{\mathrm{M}}$ and $\mathrm{L}$, with one exception of the effect of $\mathrm{J} \times \mathrm{M}$ interaction on $\mathrm{L}$. Whereas, in DR, only two main effects, inter-band spacing ( $\left.\mathrm{I}^{\prime}\right)$ and jute plant density $\left(\mathrm{J}^{\prime}\right)$, had highly significant effect $(\mathrm{p}<0.05)$ on fibre yield, mungbean seed yield, $\mathrm{L}_{\mathrm{J}}$ and $\mathrm{L}_{\mathrm{M}}$. Dense and intimate planting of jute had more competitive effect on mungbean seed yield.

\section{Yield}

In sole cropping, jute fibre yield was 30.38 and $26.00 \mathrm{q} \mathrm{ha}^{-1}$ in SR and DR, respectively; and mungbean seed yield was $16.00 \mathrm{q} \mathrm{ha}^{-1}$. Sole crop recorded higher yield than in intercropping (Table 2 and 3). Thus, sole crops-maintained supremacy over intercropping systems and may be due to the absence of interactional crop competition in the sole cropping environment, balanced fertilization and limited disturbance to the habitat (Banik et al., 2000).

In SR system, jute fibre yield varied between 21.38 and $29.50 \mathrm{q} \mathrm{ha}^{-1}$ with overall mean of $25.50 \mathrm{q} \mathrm{ha}^{-1}$ and mungbean seed yield varied between 4.19 and $11.44 \mathrm{q} \mathrm{ha}^{-1}$ with overall mean of $7.28 \mathrm{q} \mathrm{ha}^{-1}$. These results conformed to an earlier limited study in jute-mungbean intercropping with alternate row planting (Ghorai et al., 2016). Uneven distribution of rainfall was mainly responsible for overall low fibre yield in SR planting. In general, jute fibre yield was low in wider spacing of $40-45 \mathrm{~cm} \times 8$ $\mathrm{cm}$, due to low plant density of jute and increased with narrower spatial arrangements. It attained maximum of $29.50 \mathrm{q} \mathrm{ha}^{-1}$ in $\mathrm{T}_{6}$. In contrast, mungbean seed yield showed a reverse trend to that of jute spacing. It was low in narrow intra-jute spacing of 5-6.5 $\mathrm{cm}$ and recorded higher seed yield with wider spacing combinations of $40-45 \mathrm{~cm}$ (row to row) $\times 8 \mathrm{~cm}$ (jute to jute in a row) $\times 12 \mathrm{~cm}$ (mung to mung in a row) for $\mathrm{T}_{18}, \mathrm{~T}_{25}$ and $\mathrm{T}_{26}$.

Yield reduced for both the crops in intercropping, in mungbean more so, though seed yield was higher in double row (DR) compared to the single row (SR) planting. In DR planting, intercropped jute fibre yield varied between $16.94-23.19 \mathrm{q} \mathrm{ha}^{-1}$ with an overall mean of $20.22 \mathrm{q} \mathrm{ha}^{-1}$ and seed yield varied between $6.38-10.31 \mathrm{q} \mathrm{ha}^{-1}$ with an overall mean of $8.49 \mathrm{q} \mathrm{ha}^{-1}$. Seed yield increased sharply with increase in intramungbean spacing. In general, fibre yield in DR system was significantly $(\mathrm{p}<0.05)$ low for lower jute plant density of $30 \mathrm{~m}^{-2}$ and for inter-band spacing of $11 \mathrm{~cm}$. Fibre yield increased as density or inter-band spacing increased, though with decelerated rates. Whereas mungbean yield did not show significant variation with changes in mungbean density levels. Seed yield was significantly $(p<0.05)$ higher for low jute 
plant density of $30 \mathrm{~m}^{-2}$. These results indicated yield trade-offs with varied intimacy and densities of the component crops.

Jute plants exhibited signs of $\mathrm{N}$ deficiency during 50-70 DAS as plants stunted and leaves turned pale yellowish-green chlorosis that subsided later on. It might be attributed to the causes of suspension of the $\mathrm{N}$ topdressings. Substantial reduction of intercropped mungbean seed yield seems to be mainly due to the shading effect of taller jute canopy at critical stage of pod maturity. Similar results on chickpea seed yield loss were recorded in wheat-chickpea (Banik et al., 2006). Better performance of mungbean had also been reported in 3:2 row arrangement maize-mungbean intercropping (Roy et al., 2015) and may be due to more light perception facilitated by grouping of low-canopy mungbean in band (Natarajan, 1990) and minimization of interspecific crop competition (Ofori and Stern, 1987).

\section{Jute equivalent yield (JEY)}

Under SR planting, JEY values were more than $39.00 \mathrm{q}^{-1}$ in $\mathrm{T}_{6}, \mathrm{~T}_{9}, \mathrm{~T}_{15}, \mathrm{~T}_{17}, \mathrm{~T}_{18}$, $\mathrm{T}_{21}, \mathrm{~T}_{25}$ and $\mathrm{T}_{26}$ having wider spacing of $35-40 \mathrm{~cm} \times 6.5-8.0 \mathrm{~cm} \times 10-12 \mathrm{~cm}$ and 45 $\mathrm{cm} \times 8 \mathrm{~cm} \times 8-10 \mathrm{~cm}$. It attained maximum for $\mathrm{T}_{18}(40,8,12)$. JEY increased in the range of 4.9 to $45.3 \%$ over sole jute fibre yield and 22.0 to $68.9 \%$ over sole mungbean seed yield. Similar trends have been reported with 1:1 row arrangement in jute-mungbean intercropping (Ghorai et al., 2016).

Under DR planting, JEY values were higher for treatments $\mathrm{T}_{5}^{\prime}$ to $\mathrm{T}_{9}^{\prime}$. Increase in JEY was in the range of 30.73 to $51.14 \%$ over sole jute fibre yield and 12.69 to $30.28 \%$ over sole mungbean seed yield. Similar trend of increased maize equivalent yield has been reported in maize-pigeonpea with 4:2 row proportion (Lingaraju et al., 2008).

JEY values in intercropping were always higher $(\mathrm{p}<0.05)$ over sole yield of either of crops, irrespective of planting arrangements (Table 2 and 3 ). The higher relative price of mungbean seeds contributed substantially to offset the decrease in intercropped jute fibre yield and outperformed the sole fibre yield and of sole seed yield. Relative price of jute fibre and mungbean seed were $1: 1.63,1.59$ and $1: 1.89$, for the years 2016, 2017 and 2018, respectively(https://farmer.gov.in/mspstatements.aspx).

\section{Land equivalent ratio (LER)}

Partial LER values for jute $\left(\mathrm{L}_{\mathrm{J}}\right)$ were always greater than that of mungbean $\left(\mathrm{L}_{\mathrm{M}}\right)$. Values varied inversely to each other and both were less than unity. Total LER values always exceeded unity in intercropping, irrespective of row arrangement (Fig. 2 and 3). 


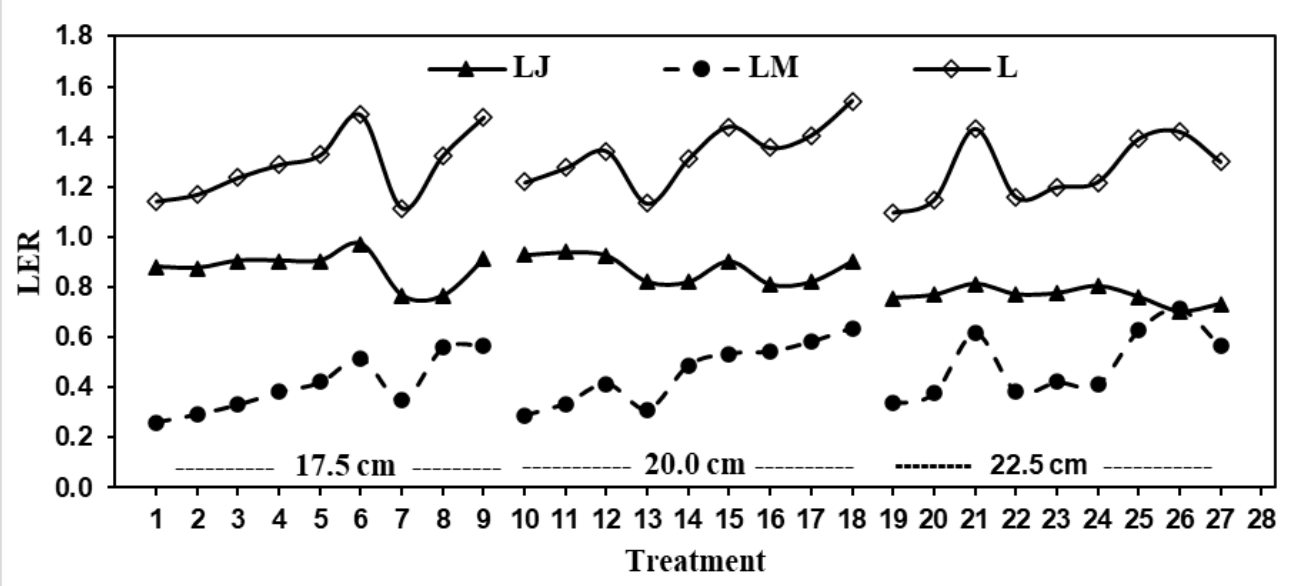

Figure 2. Land equivalent ratios at different inter-row spacing (17.5, 20.0 and 22.5 $\mathrm{cm}$ ) under altrnate single row (SR) planting as influenced by intra-row jute and mungbean spacing variations. $\mathrm{L}_{\mathrm{J}}$ and $\mathrm{L}_{\mathrm{M}}$ are partial LERs for jute and mung, respectively and $\mathrm{L}$ is the total LER

In $S R$ arrangement, $L_{J}$ values varied in the range of 0.704 to 0.971 , with an overall mean of 0.840 and $\mathrm{L}_{\mathrm{M}}$ ranged between 0.262 and 0.715 with an overall mean of 0.455. Whereas, total LER (L) for intercropping varied between 1.097 and 1.542 with an overall mean of 1.295. In general, $\mathrm{L}_{\mathrm{J}}$ values were low for low jute plant density of 28 to $30 \mathrm{~m}^{-2}$, increased with increase in jute plant density of 31 to $50 \mathrm{~m}^{-2}$, attained a maximum of 0.9712 for the plant density of $44 \mathrm{~m}^{-2}$ in $\mathrm{T}_{6}$; but decreased with further increase in density. In contrast, $\mathrm{L}_{\mathrm{M}}$ values varied reversely to that of $\mathrm{L}_{\mathrm{J}}$. These indicated that intercropping is always advantageous though components did not fully share the limiting resources. Similar results were reported in goundnut-cereal intercropping (Ghosh, 2004) and in cereal-legume intercropping (Bedoussac et al., 2015).

In DR planting, $\mathrm{L}_{\mathrm{J}}$ values varied between 0.651 and 0.892 , with an overall mean of 0.780 and $\mathrm{L}_{\mathrm{M}}$ values ranged from 0.400 to 0.645 with an average of 0.531 . Whereas, total LER (L) for intercropping varied between 1.227 and 1.412 with an overall mean of 1.311. In general, with jute plant density of $40-50 \mathrm{~m}^{-2}$ and inter-band spacing of 14 and $17 \mathrm{~cm}$, mean $\mathrm{L}_{\mathrm{J}}$ values were more than 0.79 but mean $\mathrm{L}_{\mathrm{M}}$ values were less than 0.50 . In contrast, $\mathrm{L}_{\mathrm{M}}$ values were high with low jute plant density. With $11 \mathrm{~cm}$ interband spacing, $\mathrm{L}_{\mathrm{M}}$ values were more than 0.59 with a jute plant density of $30 \mathrm{~m}^{-2}$. It indicated that by increasing jute population, intercropped fibre yield approached nearer to sole jute yield, but it reduced mungbean seed yield by $50 \%$ or more of its sole yield. Whereas, with less dense and less intimate planting of jute, $\mathrm{L}_{\mathrm{M}}$ increased. This may be due to interception of more solar radiation to mungbean and reduced inter-specific competition in alternate band planting. 


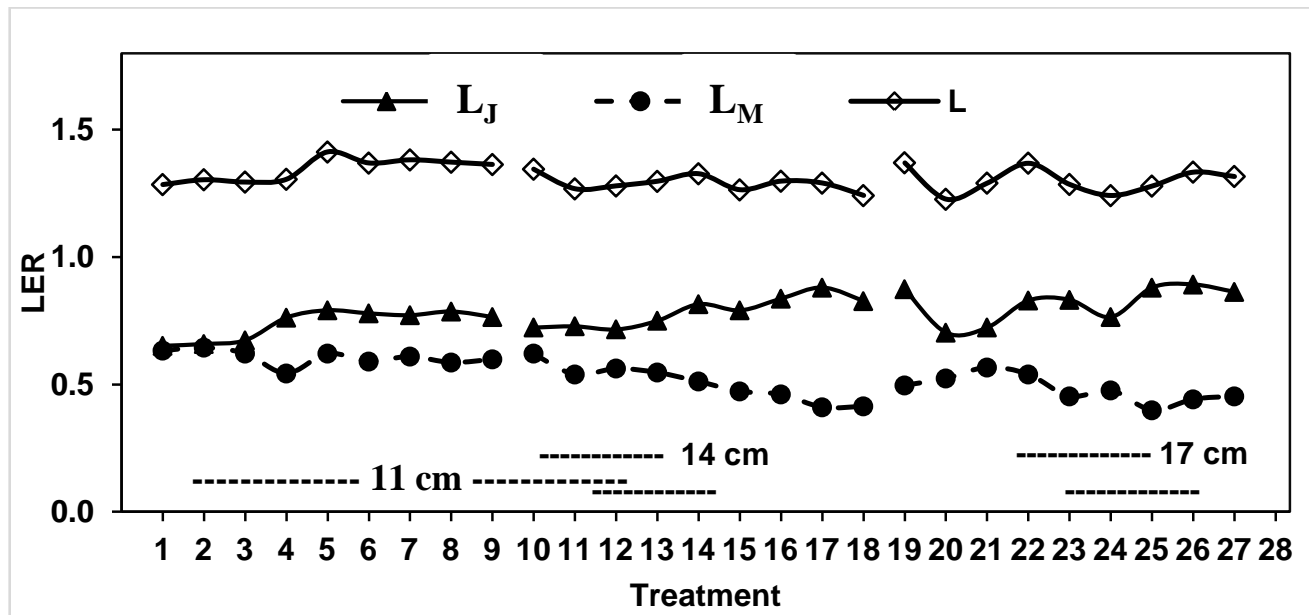

Figure 3. Land equivalent ratios at different inter-band $(11,14$ and $17 \mathrm{~cm})$ spacing under altrnate double row (DR) planting as influenced by within-row jute and mungbean spacing variations. $\mathrm{L}_{\mathrm{J}}$ and $\mathrm{L}_{\mathrm{M}}$ are partial LERs for jute and mung, respectively and $\mathrm{L}$ is the total LER

\section{Area $\times$ time equivalent ratio (ATER)}

In SR planting, with the exception of three treatments, ATER values for all the other 24 combinations exceeded unity. Whereas, in DR planting ATER values always exceeded unity. In SR planting average ATER value is 1.114 . Values were more than 1.22 for treatments $\mathrm{T}_{6}, \mathrm{~T}_{9}, \mathrm{~T}_{15}$, and $\mathrm{T}_{18}$ having $35-40 \mathrm{~cm} \times 6.5-8 \mathrm{~cm} \times 12 \mathrm{~cm}$ spacing combinations. In DR planting, ATER values ranged from 1.025 to 1.179 with a mean of 1.107 , indicating 2.5 to $17.9 \%$ advantage with jute-mungbean intercropping system over sole cropping.

\section{Competitive ability indices}

Competitive ability is a measure of the ability of one of the crops to harness limiting resources, when grown in mixtures with another component, compared to its ability to utilize those resources when grown in pure stands (Snaydon, 1991).

\section{Relative crowing coefficient $(\mathrm{K})$}

In both the row arrangements, $\mathrm{K}_{\mathrm{JM}}>1.0$ and were always higher than corresponding values of $\mathrm{K}_{\mathrm{MJ}}$. These indicated jute as highly dominant and competitive species of the two (Table 2 and 3). Combined $\mathrm{K}$ values were always $>1.0$, indicating definitive yield advantages due to intercropping. Narrower range of $\mathrm{K}$ values in DR indicated that the degree of competition in DR was lesser compared to SR system. Grouping of crops in band arrangements might have eased the intensity of competition (Natarajan, 1990). These results conformed to studies in groundnut-cereal (Ghosh, 2004) and wheat-chickpea intercropping (Baniket al., 2006). 


\section{Aggressivity (A)}

In the two row arrangements, $A_{J}$ values were always positive with one exception of $\mathrm{T}_{27}$ in SR planting. $\mathrm{A}_{\mathrm{J}}$ values increased either with the increase in jute plant density or with decrease in inter-row / inter-band spacing. In contrast, $A_{M}$ values were always negative with one exception of $T_{27}$ in SR. Positive values of $A_{J}$ indicated that jute was the dominant species. Higher $\mathrm{A}_{J}$ values indicated that the intensity of dominance increased with dense and intimate planting of jute; values lessened progressively as jute planting were thinner and less intimate; and attained the lowest value with spacing of $45 \mathrm{~cm} \times 8 \mathrm{~cm} \times 8-12 \mathrm{~cm}$, where jute plant density was at its lowest of 28 $\mathrm{m}^{-2}$.

Similarly, in DR planting, with jute plant density of $50 \mathrm{~m}^{-2}$, higher values of $\mathrm{A}_{J}$ indicated that the intensity of dominance increased with dense and intimate planting of jute. It was at its low for low jute plant density of $31 \mathrm{ha}^{-1}$ and inter-band spacing of $11 \mathrm{~cm}$.

\section{Monetary advantage index (MAI)}

The higher the MAI value, the more profitable is the cropping system (Ghosh 2004). Economic feasibility of jute-mungbean intercropping systems were analyzed using MAI. MAI values varied between 9012 and $49640 ₹$ ha $^{-1}$ with an overall mean of 27180 ₹ ha ${ }^{-1}$ in SR planting (Table 2). Monetary advantages were more than 30000 ₹ $\mathrm{ha}^{-1}$ in in 11 treatments with spacing combinations of $35-40 \mathrm{~cm} \times 6.5-8 \mathrm{~cm} \times 10-12$ $\mathrm{cm}$ and $45 \mathrm{~cm} \times 5-8 \mathrm{~cm} \times 10-12 \mathrm{~cm}$. In some of these treatments, contribution from mungbean was marginal with substantial contribution from jute fibre, or vice versa. For $\mathrm{T}_{6}, \mathrm{~T}_{9}, \mathrm{~T}_{15}$ and $\mathrm{T}_{18}$, contribution from both the crops were substantial.

In DR planting, MAI values varied between 23246 and $42415 ₹$ ha $^{-1}$ with an overall mean of $31829 ₹ \mathrm{ha}^{-1}$ (Table 3).In treatments $\mathrm{T}_{2}^{\prime}$ to $\mathrm{T}_{9}^{\prime}$, the contribution from mungbean seed yield was substantial with moderate contribution from jute fibre; whereas, for the treatments $\mathrm{T}_{10}^{\prime}, \mathrm{T}_{13}^{\prime}, \mathrm{T}_{14}^{\prime}, \mathrm{T}_{16}^{\prime}, \mathrm{T}_{19}^{\prime}, \mathrm{T}_{22}^{\prime}, \mathrm{T}_{26}^{\prime}$ and $\mathrm{T}_{27}^{\prime}$, the contributions from jute was substantial and that of mungbean was marginal. Narrow range of variation in MAI values for DR planting indicated stable benefit. These results conformed to the results of higher MAI in groundnut/cereal fodder intercropping whenever LER and K values were higher (Ghosh, 2004).

\section{CONCLUSION}

Traditional sole jute farming in tropical and subtropical areas of India and Bangladesh are facing challenges of low profitability and area shrinkage. In the wake of Green Revolution, cereals had also taken over the usual growing areas of many traditional crops including pulses along the jute tracts of these two nations and reduced mungbean area and production. Advances in knowledge and technology aided in successful realization of jute-mungbean intercropping. This study indicated that jute-mungbean intercropping is potent to address the rising concern on economic 
return and nutrition. Irrespective of 1:1 or 2:2 row arrangements, jute-mungbean intercropping system is always superior to sole cropping in productivity and economic return. Intercropping is potent to overcome profitability constraints in jute farming and encourages vertical area expansion of mungbean to secure protein nutrition to jute farmers, predominantly small and marginal farmers of India and Bangladesh. Double row planting is more advantageous to stabilize yield, income and crop management. With little prudence in crop management strategies, adoption of this intercropping system might be a potent and sustainable solution to arrest jute area decline and increase of mungbean area and production, along jute tracts of IndoBangla subcontinent. It would bring desirable protein security and numerous other benefits as well. This study would give impetus to investigate other aspects of jutemungbean intercropping.

\section{ACKNOWLEDGMENT}

The authors acknowledge the support of Director, ICAR-CRIJAF in conducting the experiments. The authors are grateful to G. Das, D. Dey and A.N. Dey for their valuable technical assistance with field plot management and data collection; Dr. D. Barman for providing weather records of the institute; and N. Paul and S. Chakraborty for their help in data entry and layout drawing.

\section{CONFLICT OF INTEREST}

The authors declare that they have no conflict of interest.

\section{REFERENCES}

Ali, M. and Gupta, S. (2012). Carrying capacity of Indian agriculture: pulse crops. Current Science, 102: 874-881.

Ali, M. and Mishra, J.S. (1993). Intercropping of blackgram (Phaseolus mungo) and greengram (P. radiata) with spring sunflower (Helianthus annus). Indian Journal of Agricultural Science, 63: 493-495.

Altieri, M.A., Funes-Monzote, F.R. and Petersen, P. (2012). Agroecologically efficient agricultural systems for smallholder farmers: contributions to food sovereignty. Agronomy for Sustainable Development, 32: 1-13.

APY Pulses (2016-17). State-wise Area, Production and Yield of all Pulses from 1997-98 to 2016-17. http://dpd.gov.in/Stats_new.htmAccessed $25^{\text {th }}$ November, 2020.

Banik, P., Midya, A., Sarkar, B.K. and Ghose, S.S. (2006). Wheat and chickpea intercropping systems in an additive series experiment: Advantages and weed smothering. European Journal of Agronomy, 24: 325-333.

Banik, P., Sasmal, T., Ghosal, P.K. and Bagchi, D.K. (2000). Evaluation of mustard (Brassica campestris Var. Toria) and legume intercropping under 1:1 and 2:1 Row-Replacement Series Systems. Journal of Agronomy and Crop Science, 185: 9-14.

Bedoussac, L., Journet, E.P., Hauggaard-Nielsen, H., Naudin, C., Corre-Hellou, G., Jensen, E.S., Prieur, L. and Justes, E. (2015). Ecological principles underlying the increase of 
productivity achieved by cereal-grain legume intercrops in organic farming. A review. Agronomy for Sustainable Development, 35: 911-935.

Chadha, M.L. (2010). Short duration mungbean: a new success in South Asia. Asia-Pacific Association of Agricultural Research Institutions. FAO RAP, Bangkok, Thailand. Pp. 55.

Dasgupta, S. and Roy, I. (2016). Proceedings of the regional consultation on the promotion of pulses in Asia for multiple health benefits. Bangkok, Thailand: FAO RAP

De Wit, C.T. (1960). On Competition. Verslagen Landbouwkundige Onderzoekigen, 66: 1-82.

George, R. (2015). The economic lives of smallholder farmers: An analysis based on household data from nine countries. FAO, Rome.

Ghorai, A.K., Kumar, M. and Kar, C.S. (2016). Weed smothering in jute with green gram intercropping. Indian Journal of Weed Science, 48: 343-344.

Ghosh, P.K (2004). Growth, yield, competition and economics of groundnut/cereal fodder intercropping systems in the semi-arid tropics of India. Field Crops Research, 88: 227237.

Hiebsch, C.K. and McCollum, E.R. (1987). Area-X-Time equivalency ratio: A method for evaluating the productivity of intercrops. Agronomy Journal, 79: 15-22.

Huxley, P.A. and Maingu, Z. (1978). Use of systematic design as an aid to the study of intercropping: some general considerations. Experimental Agriculture, 14: 49-56.

Islam, M.A. and Adjesiwor, A.T. (2018). Nitrogen fixation and transfer in agricultural production systems. In: Nitrogen in Agriculture -Updates (Editors: Amanullah and S. Fahad). Intech Open, Rijeka, Croatia. Pp. 95-110.

Kumari, K., Devegowda, S.R. and Kushwaha, S. (2018). Trend analysis of area production and productivity of jute in India. The Pharma Innovation Journal, 7: 58-62.

Lal, R.B. and Ray, S. (1976). Economics of crop production of different intensities. Indian Journal of Agricultural Sciences, 46: 93-96

Li, J., Huang, L., Zhang, J., Coulter, J.A., Li, L. and Gan, Y. (2019). Diversifying crop rotation improves system robustness. Agronomy for Sustainable Development, 39: 38.

Lingaraju, B.S., Marer, S.B. and Chandrashekar, S.S. (2008). Studies on intercropping of maize and pigeonpea under rainfed conditions in northern transitional zone of Karnataka. Karnataka Journal of Agricultural Sciences, 21: 1-3.

Mandal, A. (2016). Comparative study of marginal farms in India vis-a-vis West Bengal; Evidences from Last Decade. Economic Affairs, 61(4): 589-598.

McGilchrist, C.A. (1965). Analysis of competition experiments. Biometrics, 21: 975-985.

Mead, R. and Stern, R.D. (1980). Designing experiments for intercropping research. Experimental Agriculture, 16: 329-342.

Mead, R. (1990). Appropriate experimental designs and treatment structures for intercropping. In S.R. Waddington, A.F.E. Palmer and O.T. Edje (Eds.), Research Methods for Cereal/Legume Intercropping: Proc. of a Workshop on Res. Methods for Cereal/Legume Intercropping in Eastern and Southern Africa. CIMMYT, Mexico. Pp. 94-101. 
Natarajan, M. (1990). Spatial arrangements of the component crops in developing intercropping systems: Some concepts and methodologies. In S.R. Waddington, A.F.E. Palmer and O.T. Edje (Eds.), Research Methods for Cereal/Legume Intercropping: Proc. of a Workshop on Res. Methods for Cereal/Legume Intercropping in Eastern and Southern Africa. CIMMYT, Mexico. Pp. 68-73.

Ofori, F. and Stern, W.R. (1987). Cereal-legume intercropping systems. Advances in Agronomy, 41: 41-90.

Rahaman, M. and Prasad, S. (2018). Development of pre-mature flowering resistant tossa jute (C. olitorius L.) varieties for early sowing with specific reference to study of DNA profiles. International Journal of Bioresource Science, 5:107-116.

Rao, R.M. and Willey, R.W. (1980). Evaluation of Yield Stability in Intercropping: Studies on Sorghum/Pigeonpea. Experimental Agriculture, 16: 105-116

Roy, S., Barman, M., Puste, A. M., Gunri, S.K. and Jana, K. (2015). Growth, yield, water use efficiency and competitive functions of intercropping system of maize (Zea mays L.) and mungbean (Vigna radiata L.) as influenced by irrigation. SAARC Journal of Agriculture, 13: 94-107

Snaydon, R.W. (1991). Replacement or additive designs for competition studies. Journal of Applied Ecology, 28: 930-946.

Thattil, R.O. and Costa, W.A.M.J.de. (1988). Spacing experiment with maize $\times$ mungbean intercropping system using a '3-way' systematic design. Tropical Agriculturist (Sri Lanka), 144: 109-122.

Willey, R.W. and Rao, M.R. (1980). A competitive ratio for quantifying competition between intercropping. Experimental Agriculture, 16: 117-125.

Willey, R.W. (1979). Intercropping-its importance and research needs. Part 1. Competition and yield advantages. Field Crop Abstracts, 32: 1-10. 